\title{
Docosahexaenoic Acid (DHA) Induces P53-Dependent Growth Inhibition in Transformed Colon and Lung Cell Lines Expressing Wildtype $P 53$
}

\author{
Keith D. Kikawa*, Noah T, Ahwah SM and Pardini RS \\ Department of Biochemistry, University of Nevada, Reno
}

\begin{abstract}
Supplementation with n-3 polyunsaturated fatty acids, both in dietary in vivo studies, as well as in vitro tissue culture models, has anti-proliferative effects on tumor cells. In the current study, the role of p53-dependent growth inhibition by docosahexaenoic acid (DHA), an n-3 polyunsaturated fatty acid, is examined. Previous work has established that DHA is capable of growth inhibitory effects independent of p53 mutational status in colon carcinomas, however, one of the same studies showed an increase in the number of apoptotic cells (measured by Annexin V-FITC) only in the DHAtreated cells of the colon carcinoma with wildtype p53. To determine the potential role of p53 on the growth inhibition observed with DHA treatment of the human colon carcinomas COLO-205 (wildtype p53) and WiDr (mutant p53, His 237) and the human lung adenocarcinomas A549 (wildtype p53) and H441 (mutant p53, codon 158), p53-specific siRNA's and a chemical inhibitor of p53, pifithrin- $\alpha$, were employed in vitro. Significant increases in the number of DHA-treated cells by p53 siRNA or pifithrin- $\alpha$ addition were observed only in the COLO-205 and A549 cell lines expressing wildtype $\mathrm{p} 53$, and these correlated with a reduction in the percentage of apoptotic and necrotic cells. This data confirms a role for p53-dependent growth inhibition with DHA treatment.
\end{abstract}

\section{Introduction}

Unlike most diseases, cancer causation cannot be easily classified, as its origin usually varies from patient to patient and is often due to multiple dysfunctions (Hanahan and Weinberg, 2000). However, because of the prevalence and sizeable contribution to mortality rates worldwide, developing an understanding of mechanisms that frequently contribute to cancer incidence is of the utmost importance. Mutations in the p53 gene resulting in a loss of function are present in a high percentage of cancers, making it a widely studied facet in the causation of cancer (Sidransky and Hollstein, 1996). This makes sense, as p53 is a transcription factor that directly binds to the promoter sequences of specific target genes resulting in the regulation of cell growth and survival, making it an important regulatory component for normal cell behavior (Hainaut and Hollstein, 2000; el-Diery, 1998; Levine, 1997; Wang et al., 1995).

Nutrition can play a major role in cancer mortality with some estimating it can account for up to $30 \%$ of cancer-related deaths (Glade, 1999). Numerous epidemiological studies have looked at the influence of diet on cancer (Willett, 2002; Lanier et al., 1976). One widely studied area is the anti-cancer properties of $n-3$ polyunsaturated fatty acids (PUFAs), specifically; docosahexaenoic acid (DHA) is a 22-carbon n-3 PUFA that has been shown to significantly reduce cancer growth both in vitro and in vivo (Pardini, 2006).

While numerous mechanisms have been linked to DHA's antiproliferative effects on cancer, several reports have focused on whether p53 plays a role in DHA-induced growth inhibition (ToitKohn et al., 2009; Zand et al., 2007; Kolar et al., 2007; Kato et al., 2007; Tsujita-Kyutoku et al., 2004). DHA treatment has been shown to modify p53 protein levels in addition to levels of phosphorylation at $\operatorname{Ser}^{15}$ (Toit-Kohn et al., 2009; Zand et al., 2007). While previous studies on colon cancer models have concluded that DHA is capable of exerting growth inhibitory effects independent of p53 mutational status (Zand et al., 2007; Kolar et al., 2007), there is some evidence p53-dependent pathways may be inhibiting cell growth. In one previous study, DHA was found to increase the number of apoptotic cells only in colon cancer cells containing wildtype p53 (Kolar et al., 2007).
In the current study, the colon cancer cell lines WiDr and COLO 205, in addition to the lung cancer cell lines H441 and A549 were selected. WiDr, a derivative of the HT-29 colon adenocarcinoma, posses a mutated p53 codon (His273) (Chen et al., 1987; Tamura et al., 1995), while H441 also has a p53 mutation (Lai et al., 2000). Both COLO 205 and A549 have wildtype p53 (Kato et al., 2007; Mukhopadhyay and Roth, 1997). To determine the role of p53 in DHA-induced effects on cell proliferation, apoptosis and anchorageindependent cell growth (soft agar), pifithrin- $\alpha$, a chemical inhibitor of p53 (Komarov et al., 1999), and p53-specific siRNAs were used. The findings suggest that while DHA-induced growth inhibition and apoptosis can occur independent of p53 mutational status, cell lines expressing wildtype p53 do undergo p53-dependent cell death and growth inhibition.

\section{Materials and Methods}

\section{Cell lines, antibodies \& reagents}

A549 human lung adenocarcinoma cells, H441 human lung carcinoma, COLO 205 human colon carcinoma and WiDr human colon carcinoma cells were purchased from ATCC (Manasses, VA). Fatty acid methyl esters (FAME) (Sigma, St. Louis, MO) were dissolved in ethanol $(\mathrm{EtOH})$, flushed with nitrogen gas, protected from light and stored at $-20^{\circ} \mathrm{C}$ for no more than 60 days. Monoclonal antibodies specific to p53 (Santa Cruz Biotechnology, Santa Cruz, CA) or $\beta$-actin (Abcam, Cambridge, MA) were purchased for Western Blot analyses. Validated

*Corresponding author: Keith D. Kikawa, Department of Biochemistry, University of Nevada, Virginia St. MS330, Reno, Nevada; E-mail: kkikawa@unr.eduu

Received September 28, 2010; Accepted October 18, 2010; Published October 18,2010

Citation: Kikawa KD, Noah T, Ahwah SM, Pardini RS (2011) Docosahexaenoic Acid (DHA) Induces P53-Dependent Growth Inhibition in Transformed Colon and Lung Cell Lines Expressing Wildtype P53. J Cancer Sci Ther 3: 001-004. doi:10.4172/1948-5956.1000047

Copyright: () 2011 Kikawa KD, et al. This is an open-access article distributed under the terms of the Creative Commons Attribution License, which permits unrestricted use, distribution, and reproduction in any medium, provided the original author and source are credited. 
p53 and non-silencing (NS) siRNAs, as well as HiPerFect reagent was purchased from Qiagen (Valencia, CA).

Cell culture: A549, H441 and COLO 205 cells were maintained in RPMI-1640 supplemented with 10\% FBS (Hyclone, Logan, UT), while WiDr cells were maintained in MEM supplemented with $10 \%$ BGS (Hyclone, Logan, UT). Cells were grown as monolayers at $37^{\circ} \mathrm{C}$ in a humidified environment with $5 \% \mathrm{CO}_{2}$. A549 and $\mathrm{H} 441$ human lung cancer cell lines received $100 \mathrm{uM}$ doses of FAME, while colon cancer cell lines were administered $125 \mathrm{uM}$ doses. Linoleic acid (LA), is an $\mathrm{n}-6$ PUFA that is predominant in Western diets, and ethanol (EtOH) is used as a vehicle to dissolve FAME and serves as a control treatment for most of the experiments.

siRNA treatments: siRNA treatments were performed according to the manufacturers protocol. Briefly, siRNA (Qiagen, Valencia, CA) was placed in OptiMem (Invitrogen, Carlsbad, CA) and allowed to complex with HiPerFect (Qiagen, Valencia, CA) reagent before being added dropwise to cell cultures plated at specific densities 24 hours earlier. 24 hours after siRNA treatments, lung cultures were supplemented with 100 uM FAME of DHA and linoleic acid (LA), or an equal volume of ethanol (EtOH), while colon cell lines received $100 \mathrm{uM}$ or $125 \mathrm{uM}$ FAME or an equal volume of EtOH.

Western blot analysis: Cells were washed with ice-cold PBS and lysed using GTP-lysis buffer [50mM HEPES ( $\mathrm{pH} 7.5), 15 \mathrm{mM} \mathrm{NaCl}$, $6 \mathrm{mM}$ sodium deoxycholate, $1 \% \mathrm{NP}-40,10 \%$ glycerol, $10 \mathrm{mM} \mathrm{MgCl} 2,1$ $\mathrm{mM}$ EDTA] containing freshly added protease and phosphatase inhibitors. Samples were centrifuged at $16,000 \times g$ for 10 minutes at $4^{\circ} \mathrm{C}$. Supernatants were analyzed for protein concentration using BIO RAD's DC assay (Hercules, CA). Samples were resolved by SDS-PAGE and transferred to nitrocellulose membranes (BIO RAD, Hercules, CA) and probed with specific antibodies. Detection was performed using HRP-conjugated secondary antibodies and visualized with ECL (GE Healthcare, Buckinghamshire, UK).

Anchorage-independent cell growth assays: A $0.4 \%$ solution of noble agar (Sigma, St Louis, MO) was combined with RPMI-1640 media containing $25 \mathrm{uM}$ concentrations of FAME or EtOH. The cells were seeded over a $0.8 \%$ noble agar base layer in triplicate for each independent experiment. Cells were rehydrated every 3 days with the appropriate medium. Cells were rehydrated twice weekly with RPMI1640 media without additional fatty acid or siRNA treatments. After 3 weeks, cells were stained with crystal violet (Sigma, St. Louis, MO) and colonies numbers were determined.

Annexin V-FITC/PI analysis: Analysis was performed as described previously (Tamura et al., 1995). Briefly, cells were trypsinized, counted, and $1.0 \times 10^{6}$ cells were assayed using an Annexin V-FITC Apoptosis Detection Kit (Calbiochem, San Diego, CA). Briefly, cells were added to binding buffer and incubated with Annexin V-FITC, washed, resuspended and had propidium iodide added before being analyzed by flow cytometry.

Statistical analysis: All experimental results were independently repeated at least three times. All quantitative data shown represent the compiled data as percentages versus control treatments with error bars representing standard deviation, and statistical analyses were performed with ANOVA using the Tukey method for pairwise comparison, with values of at least $\mathrm{p} \leq 0.05$ being considered significant unless stated otherwise.

\section{Results}

p53 Inhibition in cancer cells with wildtype p53 partially reverse DHA-induced growth inhibition

To inhibit wildtype p53 function, pifithrin- $\alpha$ treatments and concentrations of siRNA specific to p53 were optimized for each cell line (data not shown). For this experiment, cells received either fatty acid methyl esters (FAME) of the n-3 PUFA docosahexaenoic acid (DHA), the n-6 PUFA linoleic acid (LA), or ethanol (EtOH), in addition to no treatment (NT), mock transfection with HiPerFect reagent alone (Mock), control non-silencing siRNA (NS), p53-specific siRNA (p53), or pifithrin- $\alpha$ (Pifithrin). A sample blot of A549 cells treated with p53 siRNA is shown to verify the efficacy with siRNA treatment (Figure 1). It is worthwhile to note that DHA treatments do appear to slightly increase p53 protein expression, most notably for the expose shown in the p53 siRNA treatment (Figure 1). This is consistent with earlier reports that demonstrate DHA is capable of modifying p53 protein expression (Toit-Kohn et al., 2009; Zand et al., 2007).

In A549 cells that possess wildtype p53, the inhibition of cell proliferation was partially reversed from $63.66 \pm 5.30 \%$ to $91.24 \pm$

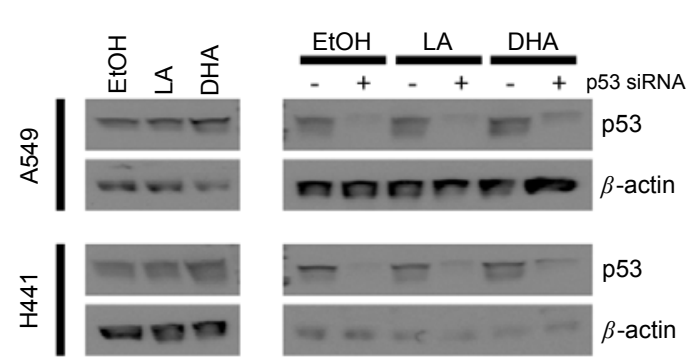

Figure 1: A549 lung cancer cells were treated with $100 \mu \mathrm{M}$ of linoleic acid (LA), docosahexaenoic acid (DHA), or ethanol (EtOH), or $25 \mathrm{nM}$ of control/nonsilencing siRNA (NS siRNA), or siRNA specific to p53 (p53 siRNA). 24 hours later siRNA treatments were supplemented with $100 \mu \mathrm{M}$ of LA, DHA, or EtOH. 48 hours after PUFA supplementation, cells were processed, resolved by SDS$\mathrm{PAGE}$, transferred to nitrocellulose membranes and probed with antibodies specific to $p 53$ and $\beta$-actin protein.

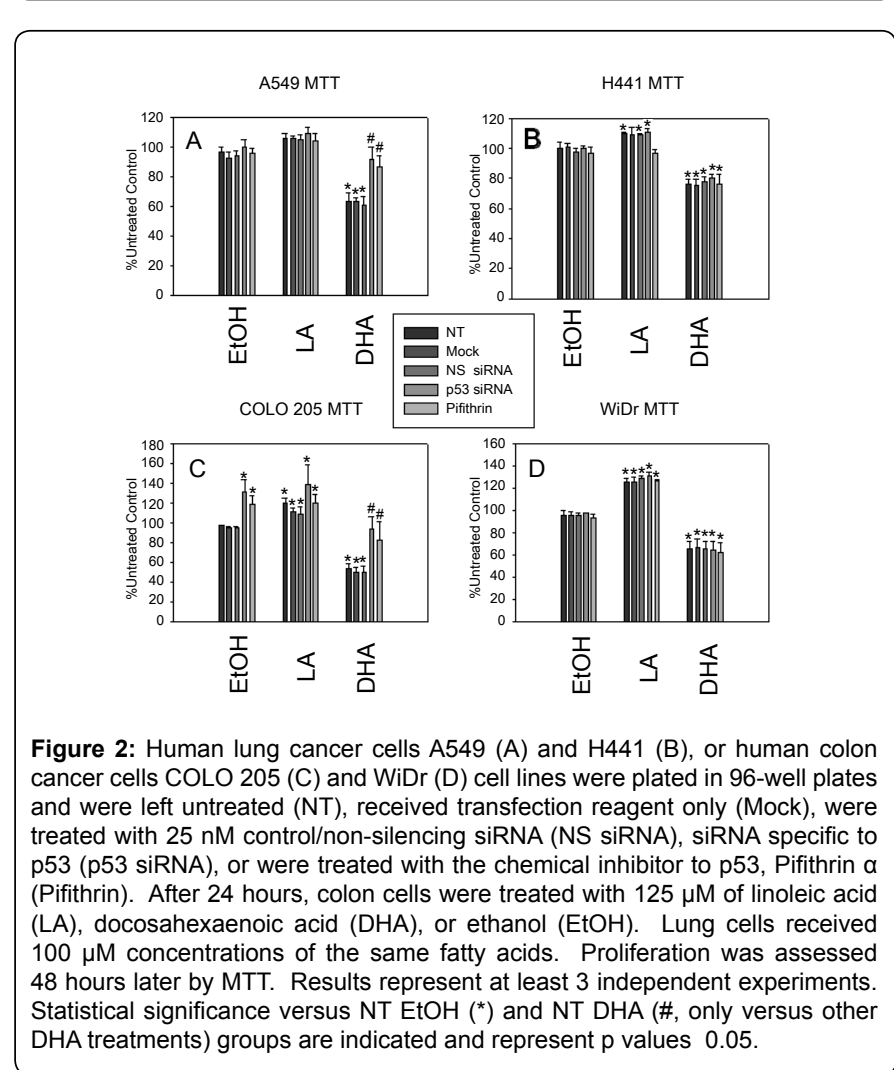


$8.79 \%$ with p53 siRNA and to $86.40 \pm 7.80 \%$ with pifithrin- $\alpha$ (Figure 2A). Similarly, COLO 205 cells expressing wildtype p53 saw growth inhibition somewhat inverted by the addition of siRNA (93.81 \pm $11.82 \%)$ and pifithrin- $\alpha(82.93 \pm 17.81 \%)$ (Figure $2 \mathrm{C})$. Interestingly, the control EtOH group of the COLO 205 cells treated with p53 inhibitors also showed significant enhancement in cell proliferation, with siRNA $(131.19 \pm 11.89 \%)$ and pifithrin- $\alpha(118.63 \pm 8.57 \%)$ both showing increases (Figure 2C). Neither of the cell lines expressing mutant p53 demonstrated any significant differences in response to the p53 inhibitors (Figures 2B and 2D). Taken together, these data demonstrate that there is p53-dependent inhibition on cell proliferation due to DHA supplementation occurring in A549 and COLO 205 cells expressing wildtype p53.

Changes in cell proliferation due to DHA supplementation correlate with increases in apoptosis/necrosis and are partially reversed by $\mathrm{p} 53$ inhibition

To determine how effects on cell proliferation might relate to cell death by apoptosis and/or necrosis, cells receiving DHA treatment in combination with p53 inhibitors were analyzed for increases in the number of apoptotic/necrotic cells. For these experiments, only the lung cancer cell lines were used. Irrespective of p53 status, DHA treatment increased the percentage of apoptotic and necrotic cells in both A549 cells with wildtype p53 (from $5.78 \pm 1.72 \%$ to $38.06 \pm$ $6.05 \%$ ) and $\mathrm{H} 441$ cells with mutant p53 (from $3.87 \pm 0.86 \%$ to 38.74 $\pm 4.91 \%$ ) (Figure 3 ). However, upon the addition of p53 inhibitors, only A549 cells saw significant reductions in cell death with siRNA treatments reducing the percentage to $19.76 \pm 5.29 \%$ and pifithrin- $\alpha$ treatments dropping to $27.37 \pm 3.76 \%$ (Figure 3 ). These results indicate that the p53-dependent effects noted in DHA treatments relate in part to cell death, most likely due to the induction of apoptosis.

\section{Inhibition of p53 results in increased anchorage-independent} cell growth in A549 cells

Because standard tissue culture models are not always indicative of how cells behave in vivo, in vitro soft agar assays were performed to determine how the previous findings might relate to results in more translational models. Interestingly, unpublished data from our laboratory has shown IC50 concentrations of DHA are less than $1 / 4^{\text {th }}$ the amount when examining colony formation and anchorage-

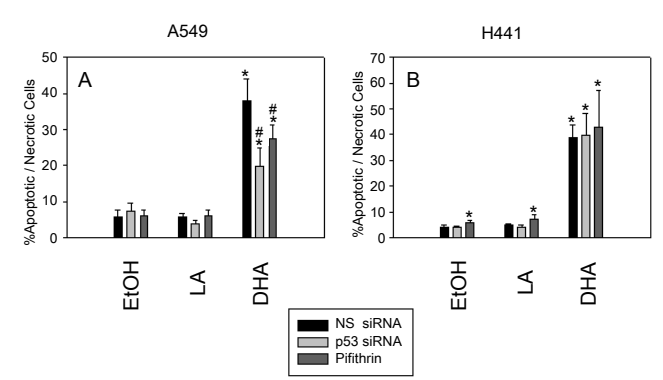

Figure 3: A549 \& H441 human lung cancer cells were treated with $25 \mathrm{nM}$ of control/non-silencing siRNA (NS siRNA), siRNA specific to p53 (p53 siRNA), or the chemical inhibitor to p53 Pifithrin (Pifithrin). 24 hours later they were supplemented with $100 \mu \mathrm{M}$ of linoleic acid (LA), docosahexaenoic acid (DHA), or ethanol (EtOH). 48 hours after PUFA supplementation, cells were analyzed with an Annexin V FITC Apoptosis Detection Kit (Calbiochem®) by flow cytometry. The \% Apoptotic/Necrotic cells were quantified from the gated population of cells in the upper and lower right quadrants. Statistical significance versus NS siRNA EtOH $\left(^{*}\right)$ and NS siRNA DHA (\#, only versus other DHA treatments) groups are indicated and represent $p$ values $\leq 0.05$
A

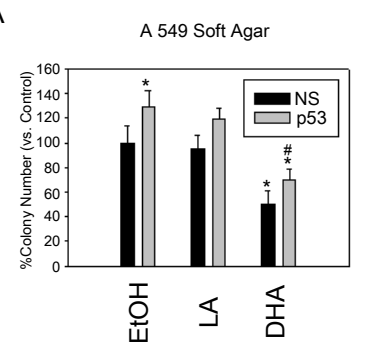

B
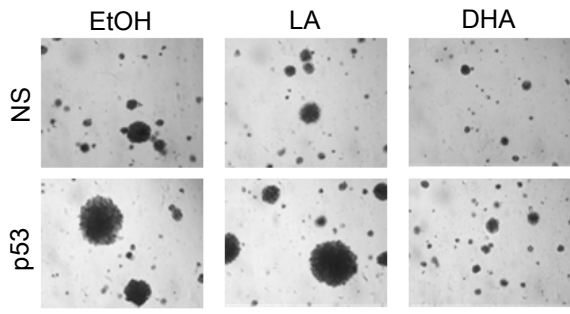

Figure 4: A549 lung cancer cells were treated with $25 \mathrm{nM}$ of control/nonsilencing siRNA (NS siRNA), siRNA specific to p53 (p53 siRNA) and suspended in an RPMI/Soft agar solution over a basement layer. Plates were allowed to grow for 2 weeks and were rehydrated every 3 days before being stained with crystal violet before being counted $(A)$ and photographed $(B)$. Statistical significance versus NS siRNA EtOH $\left(^{*}\right)$ and NS siRNA DHA (\#, only versus other DHA treatments) groups are indicated and represent $p$ values $\leq 0.05$.

independent growth in soft agar compared to standard tissue culture models. When p53 siRNA treatments were used on A549 cells in combination with EtOH, LA and DHA supplementation, colony formation increased in all cases (Figure 4A). Images of representative fields are also provided for reference (Figure 4B). With all of the enhancements in colony formation showing significance, DHA treatments did not appear to respond any better to p53 siRNA treatment than control EtOH and LA supplementations.

\section{Discussion}

This study adds new information on DHA-induced inhibition of cell proliferation by demonstrating a p53-dependent effect in lung and colon cancer cells expressing wildtype p53. Both p53-specific siRNA and pifithrin- $\alpha$, a chemical inhibitor of p53 (Komarov et al., 1999), result in a partial, but significant reversal of decreased cell proliferation upon DHA supplementation in cell lines expressing wildtype p53. These alterations in cell growth correlated with decreased percentages in the number of apoptotic and necrotic cells. These data confirm previous findings that suggest some of the effects of DHA may be p53-dependent in cell lines expressing wildtype p53. However, when anchorage-independent growth in soft agar was examined, inhibiting p53 did not have a preferential effect in the DHA-treated group, conferring greater colony numbers in control treatments as well. This information suggests that the ability of DHA to inhibit colony formation in soft agar may be unrelated to its p53dependent effects on cell growth in standard tissue culture models, thus bringing into question how relevant these findings might be in an in vivo model.

While numerous studies have reported DHA supplementation is capable of inhibiting cell proliferation in a wide range of in vitro cancer cell models (Pardini, 2006) to our knowledge, this is the first time the effect of p53-specific siRNA, and a chemical inhibitor of p53 has been used. An earlier study did employ a genetically matched model of human colon cancer that appeared to show a higher apoptotic index for DHA in cells with wildtype p53, though in this 
model, the apoptotic index noted with DHA was no different than what was seen for LA regardless of p53 status (Kolar et al., 2007). It is important however to remember that the effect of DHA has been linked to a number of different mechanisms (Pardini, 2006) and can be highly cell line-dependent (Calviello et al., 2004), and our data assessment combined both apoptotic and necrotic cells.

Understanding how n-3 PUFAs exert their anti-cancer effects is of critical importance, as nutritional supplements provide a promising opportunity to enhance conventional chemotherapeutics without the undesirable side effects often associated with these agents. While it is clear DHA can exhort inhibitory effects independent of p53 status, it also seems apparent that in some cell models there may be a p53dependent response.

\section{Acknowledgements}

We would like to thank Eastern Star for their generous donations.

\section{References}

1. Hanahan D, Weinberg RA (2000) The hallmarks of cancer. Cell 100: 57-70

2. Sidransky D, Hollstein M (1996) Clinical implications of the p53 gene. Annu Rev Med 47: 285-301.

3. Hainaut P, Hollstein M (2000) p53 and human cancer: the first ten thousand mutations. Adv Cancer Res 77: 81-137.

4. el-Diery WS (1998) Regulation of p53 downstream genes. Semin Cancer Biol 8: 345-357.

5. Levine AJ (1997) p53, the cellular gatekeeper for growth and division. Cell 87 323-331.

6. Wang XW, Yeh H, Schaeffer L, Roy R, Moncollin V, et al. (1995) p53 modulation of TFIIH-associated nucleotide excision repair activity. Nat Genet 10: 188-195.

7. Glade MJ (1999) Food, nutrition, and the prevention of cancer: a global perspective. American Institute for Cancer Research/ World Cancer Research Fund, American Institute for Cancer Research, 1997. Nutrition 15: 523-526.

8. Willett WC (2002) Diet and cancer. Oncologist 5: 393-404.

9. Lanier AP, Bender TR, Blot WJ, Fraumeni JF Jr, Hurlburt WB (1976) Cancer incidence in Alaska natives. Int J Cancer 18: 409-412.
10. Pardini RS (2006) Nutritional intervention with omega-3 fatty acids enhances tumor response to anti-neoplastic agents. Chem Biol Interact 162: 89-105.

11. Toit-Kohn JL, Louw L, Engelbrecht AM (2009) Docosahexaenoic acid induces apoptosis in colorectal carcinoma cells by modulating the PI3 kinase and p38 MAPK pathways. J Nutr Biochem 20: 106-114

12. Zand H, Rhimipour A, Bakhshayesh M, Shafiee M, Nour Mohammadi I, et al (2007) Involvement of PPAR-gamma and p53 in DHA-induced apoptosis of Reh cells. Mol Cell Biochem 304: 71-77.

13. Kolar SS, Barhoumi R, Callaway ES, Fan YY, Wang N, et al. (2007) Synergy between docosahexaenoic acid and butyrate elicits p53-independent apoptosis via mitochondrial $\mathrm{Ca}^{2+}$ accumulation in colonocytes. Am J Physiol Gastrointest Liver Physiol 293: G935-G943.

14. Kato T, Kolenic N, Pardini RS (2007) Docosahexaenboic acid (DHA), a primary tumor suppressive omega-3 fatty acid, inhibits growth of colorectal cancer independent of p53 mutational status. Nutr Cancer 58: 178-187.

15. Tsujita-Kyutoku M, Yuri T, Danbara N, Senzaki H, Kiyozuka Y, et al. (2004) Conjugated docosahexaenoic acid suppresses KPL-1 human breast cancer cell growth in vitro and in vivo: potential mechanisms of action. Breast Cancer Res 6: R291-R299.

16. Chen TR, Drabkowski D, Hay RJ, Macy M, Peterson W Jr (1987) WiDr is a derivative of another colon adenocarcinoma cell line, HT-29. Cancer Genet Cytogenet 27: 125-134

17. Tamura T, Aoyama N, Saya H, Haga H, Futami S, et al. (1995) Induction of Fas-mediated apoptosis in p53-transfected human colon carcinoma cells. Oncogene 11: 1939-1946.

18. Lai SL, Perng RP, Hwang J (2000) p53 gene status modulates the chemosensitivity of non-small cell lung cancer cells. J Biomed Sci 7: 64-70.

19. Mukhopadhyay T, Roth JA (1997) Induction of apoptosis in human lung cance cells after wild-type p53 activation by methoxyestradiol. Oncogene 14: 379384

20. Komarov PG, Komarova EA, Kondratov RV, Christov-Tselkov K, Coon JS, et al. (1999) A chemical inhibitor of p53 that protects mice from the side effects of cancer therapy. Science 285: 1733-1737.

21. Calviello G, Di Nicuolo F, Gragnoli S, Piccioni E, Serini S, et al. (2004) n-3 PUFAs reduce VEGF expression in human colon cancer cells modulating the COX-2/PGE2 induced ERK-1 and -2 and HIF-1 alpha induction pathway. Carcinogenesis 25: 2303-2310. 